\title{
RISK FACTORS FOR THE DEVELOPMENT OF BRONCHIOLITIS OBLITERANS SYNDROME AFTER LUNG TRANSPLANTATION
}

Timothy J. Kroshus, MD

Vibhu R. Kshettry, MD

Kay Savik, MS

Ranjit John, MD

Marshall I. Hertz, MD

R. Morton Bolman III, MD
Objective: This study identifies specific clinical and immunologic factors in lung transplant recipients that influence the subsequent development of chronic allograft dysfunction. Methods: The study group consisted of 132 consecutive patients who received lung allografts (76 single, 25 bilateral single, and 31 heart-lung) and survived at least 90 days. One hundred twenty-one patients were used in the analysis that modeled time to development of histologic obliterative bronchiolitis or bronchiolitis obliterans syndrome. Results: Variables noted to have an effect on the time to development of bronchiolitis obliterans syndrome included cytomegalovirus pneumonitis $(R R=3.2, p=0.001)$, late acute rejection $(R R=1.3, p=0.02)$, human leukocyte antigen mismatches at the $A$ loci $(\mathbf{R R}=1.8, p=0.02)$, total human leukocyte antigen mismatches $(\mathbf{R}=$ $1.4, p=0.04)$, and absence of donor antigen-specific hyporeactivity (52\% vs $100 \%$ survival free from bronchiolitis obliterans syndrome at 2 years; $p=0.005$ ). Cytomegalovirus pneumonitis had a significant effect on time to obliterative bronchiolitis $(R R=3.6, p=0.0005)$, as did donor antigen-specific hyporeactivity $(52 \%$ vs $100 \%$ survival free from obliterative bronchiolitis at 2 years; $p=0.01$ ). In multivariate analysis, cytomegalovirus pneumonitis $(R R=3.2, p=0.02)$, human leukocyte antigen mismatches at the $A$ loci $(R R=2.4, p=0.006)$, and late acute rejection $(R R=1.3, p=0.02)$ were identified as predictors of bronchiolitis obliterans syndrome. Cytomegalovirus pneumonitis was associated with time to development of histologic obliterative bronchiolitis $(R R=2.3, p=0.02)$. Conclusions: Several risk factors were associated with the development of chronic allograft dysfunction, which, in turn, had a significant impact on long-term survival. Early identification of lung allograft recipients with risk factors for the development of bronchiolitis obliterans syndrome may allow modification in immunosuppression and antiviral therapy to potentially decrease the prevalence of this disorder. (J Thorac Cardiovasc Surg 1997;114:195-202)
Unsign hronic lung dysfunction has emerged as the most significant long-term complication after lung transplantation. ${ }^{1,2}$ Chronic lung dysfunction was defined by criteria established for patients with bronchiolitis obliterans syndrome (BOS). ${ }^{3}$ BOS is

From the Departments of Surgery and Pulmonary Medicine, University of Minnesota, Minneapolis, Minn.

Received for publication Feb. 5, 1996; revisions requested March 28, 1996; revisions received Feb. 25, 1997; accepted for publication March 27, 1997.

Address for reprints: R. Morton Bolman III, MD, Department of Surgery, University of Minnesota, Box 207 UMHC, 420 Delaware St. SE, Minneapolis, MN 55455.

Copyright (C) 1997 by Mosby-Year Book, Inc.

$0022-5223 / 97 \$ 5.00+0 \quad \mathbf{1 2} / \mathbf{1} / \mathbf{8 2 3 6 0}$ presumed to be due to chronic allograft rejection and is characterized clinically by a progressive decline in pulmonary function with the presence or absence of pathologic evidence of obliterative bronchiolitis (OB). OB is frequently unresponsive to medical therapy, with retransplantation being the only therapeutic option for many patients. An improved understanding of the pathogenesis of $\mathrm{OB}$ is essential to design rational approaches for its prevention and treatment. The mechanisms and predisposing factors leading to the development of $\mathrm{OB}$ are presently not fully clear. ${ }^{4}$ To identify possible risk factors for chronic allograft dysfunction, we used univariate and multivariate analyses to compare the demographic, immunologic, and clinical character- 
istics of patients after lung or heart-lung transplantation who have BOS and those who have stable graft function.

\section{Methods}

Patient population. A retrospective review of 149 consecutive patients undergoing pulmonary transplantation from May 1986 to January 1995 was conducted to determine the survival rate free from the development of chronic allograft dysfunction in the posttransplantation period. One hundred thirty-two patients met the study criteria, surviving at least 3 months after transplantation: 31 received heart-lung transplants, 25 bilateral sequential lung transplants, and 76 single lung transplants. Complete data were available to model time to OB or BOS status on 88 of the 132 patients; in 121 patients all data were available except HLA matching. Operative techniques used have previously been reported. ${ }^{5}$

Criteria for diagnosis of BOS and $\mathrm{OB}$. The staging of BOS was based on criteria defined for pulmonary function data and histopathology from transbronchial lung biopsy results obtained in the posttransplantation period. ${ }^{3}$ For the purpose of this analysis, two end points were defined. OB was defined as histologically proved $\mathrm{OB}^{6}{ }^{6} \mathrm{BOS}$ was defined as a BOS grade of 2 or 3 .

Immunosuppression. Standard induction triple immunosuppression therapy was instituted in the immediate preoperative period. The drugs consisted of cyclosporine ( 3 to $6 \mathrm{mg} / \mathrm{kg}$; INN: ciclosporin) and azathioprine $(2.5$ $\mathrm{mg} / \mathrm{kg}$ ), as well as perioperative methylprednisolone (500 $\mathrm{mg}$ ) immediately before graft perfusion followed by 125 mg every 8 hours for three doses thereafter. Before 1990, oral steroids were withheld for 14 days after transplantation in lung allograft recipients after the perioperative administration of methylprednisolone. An oral steroid taper was then initiated. From 1990 to the present time this taper was started immediately after perioperative coverage consisting of prednisone at $0.5 \mathrm{mg} / \mathrm{kg}$ per day and tapering to $0.1 \mathrm{mg} / \mathrm{kg}$ per day by 3 to 6 months.

Infectious disease prophylaxis. Prophylactic antibacterial agents were given in the perioperative period. All patients received trimethoprim/sulfamethoxazole twice weekly orally or pentamidine by aerosol inhalation monthly for Pneumocystis carinii prophylaxis. In the presence of donor or recipient CMV seropositivity, the following evolution in prophylaxis has occurred. CMV prophylaxis was first initiated in 1989, consisting of acyclovir (INN: aciclovir) at $800 \mathrm{mg}$ four times a day. Between December 1991 and December 1992, patients received ganciclovir $5 \mathrm{mg} / \mathrm{kg}$ twice daily for 14 days, followed by 5 $\mathrm{mg} / \mathrm{kg}$ per day for 8 weeks. From January 1993 all patients received ganciclovir $5 \mathrm{mg} / \mathrm{kg}$ twice daily for 14 days followed by $5 \mathrm{mg} / \mathrm{kg}$ per day for 8 weeks or $5 \mathrm{mg} / \mathrm{kg}$ three times a week for 8 weeks.

Management of acute rejection. Acute rejection episodes were managed with intravenous bolus doses of methylprednisolone (500 to $1000 \mathrm{mg}$ ) for 3 days followed by an oral steroid taper. Patients with steroid-resistant rejection were treated with antithymocyte globulin for 7 to 10 days or with methotrexate.
CMV pneumonitis. The diagnosis of CMV was divided into two categories (1) CMV pneumonitis-a new pulmonary infiltrate, fever, cough, dyspnea, and new CMV culture positivity or cytologic evidence of typical CMV inclusions on bronchoalveolar lavage (BAL) or histopathologic study and (2) asymptomatic CMV infection-isolation of CMV from BAL in the absence of clinical signs or symptoms. Only CMV disease before the development of BOS was used in this analysis.

Donor antigen-specific hyporeactivity (DASH) test. Peripheral blood samples were obtained from 29 lung transplant recipients 1 year after transplantation. A mixed lymphocytotoxicity assay was used to identify donor antigen-specific hyporeactivity (DASH) with homozygous typing cells as stimulator cells and recipient cells as responder cells, as previously described. ${ }^{7}$ Hyporeactivity was defined as a decreased response to stimulation by specific typing cells that define donor antigens, whereby response to homozygous typing cells defining third-party antigens remained unchanged.

Primed lymphocyte test (PLT). The primed lymphocyte test (PLT) is a method used to detect the lymphocytedefined determinants associated with the MHC antigens. ${ }^{4}$ The principle of the PLT technique is to generate responder cells against disparities expressed by the stimulator cell by incubating the cells together for 10 days. These primed cells, presumably memory cells, respond in an accelerated manner, that is, secondary response, when restimulated by cells from the original stimulator or other cells that share stimulatory determinants with the sensitizing stimulating cell.

Statistical analysis. The impact of various predictive factors on time to development of BOS or $\mathrm{OB}$ was assessed by means of proportional hazards regression., ${ }^{8} 9$ Proportional hazards regression was chosen for assessing predictors of time to BOS and OB because it does not require the specification of a probability distribution for the underlying survival times. The proportional hazards regression model specifies that the hazard for an individual person is a product of an underlying hazard function multiplied by a factor depending on the set of covariates for that patient. It also allows for the incorporation of covariates that may change value over the period of observation. $^{10}$

Proportional hazards regression generates risk ratios (RR) for each covariate in the model. These RRs reflect the increase $(R R>1)$ or decrease $(R R<1)$ in probability of BOS for each one-unit increase in the covariate. In the multivariate model, the RRs for a specific covariate are adjusted for the effects of the other covariates in the model. Analyses were performed by means of SAS and SPSS (SAS Institute, Inc., Cary, N.C.; SPSS, Inc., Chicago, Ill.).

Four time-dependent covariates were considered before diagnosis of BOS or OB: CMV pneumonitis, counts of acute rejection, counts of late acute rejection more than 3 months after transplantation, and a positive PLT result. At each specific failure time, status of CMV pneumonitis or PLT was reassigned from negative to positive if a status change had occurred since the last failure time. For cumulative counts of rejection, the status was incremented 
by as many episodes of rejection as had occurred since the last failure time.

Even though patients were included in this assessment only if they had survived 90 days or longer, it was decided to use the date of transplantation as time zero, recognizing that survival and survival free from BOS or OB would be $100 \%$ at 90 days. Because no events occurred in this period, the fact that the starting point was transplantation rather than 90 days had no real effect on the model. This was done for two reasons. First, transplantation is a natural starting point for follow-up. Second, beginning follow-up at 90 days would require several possible risk factors to be captured as two variables, one fixed and one time-dependent, making interpretation even more complex. For example, CMV pneumonitis developed before 90 days in some patients and after 90 days in others. Thus two variables for CMV pneumonitis would have been needed for each subject, one fixed that captured CMV status before 90 days and one time-dependent that captured CMV status after 90 days.

Two types of modeling were done. One set of models was based on what clinicians believed were predictive factors for development of BOS or OB based on practice. Another set of models was based on a stepwise regression analysis, with the use of the variables specified in the clinical model. Variables whose status changed over time after transplantation (CMV pneumonitis, PLT, cumulative episodes of acute rejection, and late acute rejection) were handled as time-dependent covariates. PLT and DASH were not included in the multivariate analysis because of the small number of subjects with these values.

The impact of chronic lung dysfunction on survival was also assessed by means of a proportional hazards regression, with development of BOS or OB as a time-dependent covariate. This was done to confirm, with our specific patient group, the widely known association between development of BOS or OB and increased mortality. To illustrate the effect of BOS on survival, we generated three Kaplan-Meier curves ${ }^{11}$ on subgroups of patients defined by specific time points: (1) those who survived beyond 1 year and in whom BOS was diagnosed before 1 year versus those who had survived longer than 1 year and in whom BOS had not been diagnosed by 1 year; (2) those who survived beyond 2 years and in whom BOS was diagnosed before 2 years versus those who had survived longer than 2 years and in whom BOS had not been diagnosed by 2 years; (3) those who survived beyond 3 years and in whom BOS was diagnosed before 3 years versus those who had survived longer than 3 years and in whom BOS had not been diagnosed by 3 years.

\section{Results}

Patient population and survival. The demographic, clinical, and survival characteristics for the 121 patients in this study are summarized in Table I and Fig. 1. Patients with BOS demonstrated a reduction in survival $(\mathrm{RR}=7.2, p=0.0001)$. Fig. 1 illustrates survival for patients with BOS who have reached certain time points versus those patients in whom BOS has not developed by these time points.
Table I. Demographic characteristics of lung allograft recipients $(n=121)$

\begin{tabular}{lc} 
Age (yr) & $42 \pm 13$ \\
Sex & \\
Male & 50 \\
Female & 71 \\
Primary disease & \\
Primary pulmonary hypertension & 24 \\
Eisenmenger's syndrome & 12 \\
$\alpha_{1}$-Antitrypsin deficiency & 30 \\
Chronic obstructive pulmonary disease & 29 \\
Idiopathic pulmonary fibrosis & 9 \\
Others & 17 \\
Transplant type & \\
Heart-lung & 28 \\
Bilateral single lung & 22 \\
Single lung & 72 \\
Ischemic time (min) & $252 \pm 102$ \\
\hline
\end{tabular}

Survival was also significantly different for patients with $\mathrm{OB}$ compared with those without $\mathrm{OB}(\mathrm{RR}=$ $5.3, p=0.0001$ )

Initial analysis. Recipient primary disease, type of transplant, age, ischemic time, CMV pneumonitis, PLT, total cumulative counts of acute rejection, counts of rejection episodes before 90 days, cumulative counts of late rejection after 90 days, and treatment regimens were evaluated as risk factors for the development of chronic lung dysfunction. Results are summarized in Tables II and III.

Age was dichotomized into two major groups ( $\geq 50$ years and $<50$ years) on the basis of an association with $\mathrm{OB}$ observed in a previous study. ${ }^{12}$ With this larger patient sample, this association was no longer significant. No significant difference between groups was noted in primary disease, donor organ ischemic time, steroid treatment, sex, time on the waiting list before transplantation, or follow-up.

CMV pneumonitis. The development of symptomatic CMV pulmonary infection before diagnosis of BOS was a significant risk factor for development of BOS (RR $=3.2, p=0.0001)$ and for the development of $\mathrm{OB}(\mathrm{RR}=3.6, p=0.0005)$. The effect of the three CMV prophylaxis protocols on time to development of CMV pneumonitis was assessed as a possible confounding factor. There was no significant effect of the two later protocols compared with the first protocol on time to $\mathrm{CMV}$ pneumonitis $(\mathrm{RR}=1.1, p=0.95$; and $\mathrm{RR}=1.0$, $p=0.99$, respectively). Asymptomatic CMV had no effect on time to BOS or OB.

Acute rejection. Episodes of acute rejection were looked at in two phases. Number of episodes of 

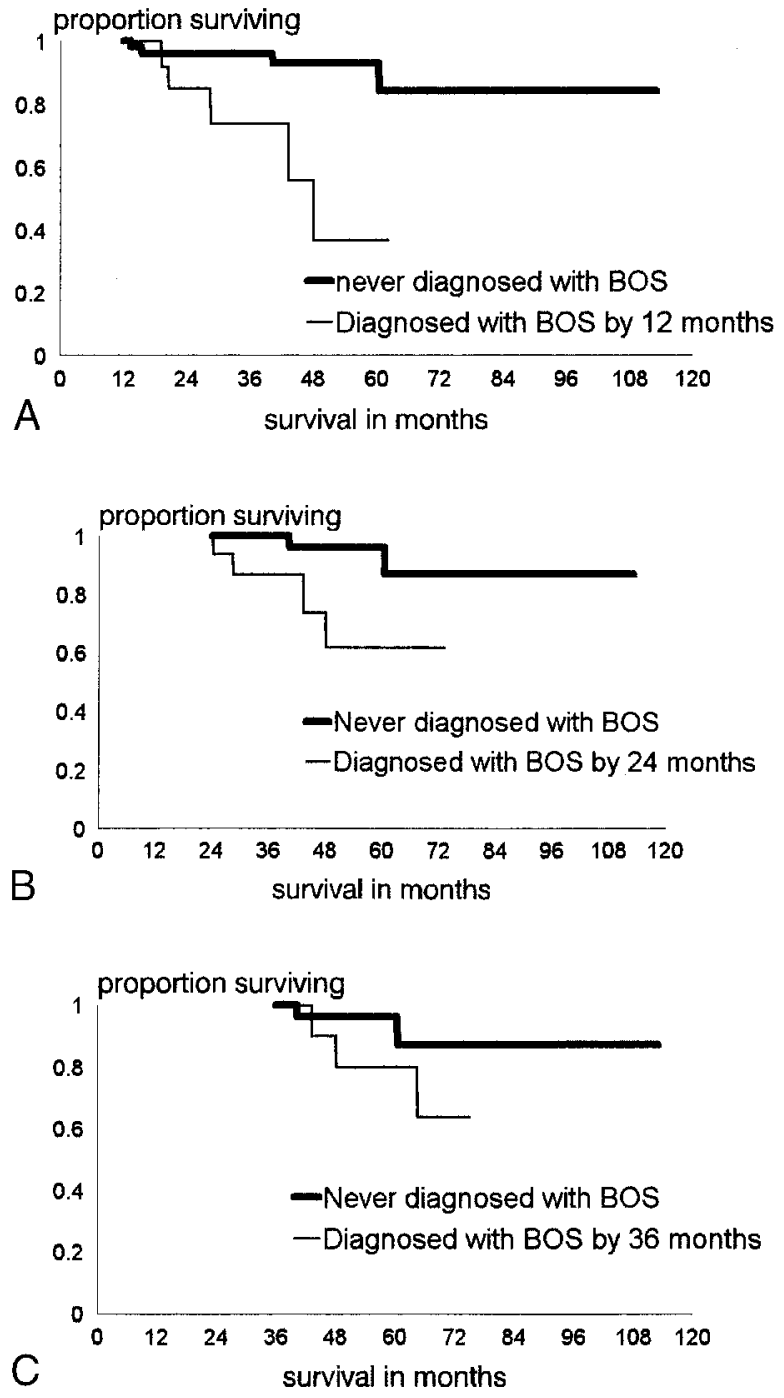

Fig. 1. A, Cumulative survival beyond 12 months for patients in whom BOS was diagnosed by 12 months compared with those patients in whom BOS was not diagnosed by 12 months. B, Cumulative survival beyond 24 months for patients in whom BOS was diagnosed by 24 months compared with those patients in whom BOS was not diagnosed by 24 months. C, Cumulative survival beyond 36 months for patients in whom BOS was diagnosed by 36 months compared with those patients in whom BOS was not diagnosed by 36 months.

acute rejection before 90 days was not significantly associated with time to development of BOS (RR = $1.2, p=0.12)$ or $\mathrm{OB}(\mathrm{RR}=1.0, p=0.83)$. Cumulative episodes of late acute rejection were significantly associated with time to development of $\mathrm{BOS}(\mathrm{RR}=1.3, p=0.02)$. In contrast, the development of $\mathrm{OB}$ was not associated with cumulative episodes of late acute rejection ( $\mathrm{RR}=0.8, p=$ $0.55)$. The effect on time to BOS or OB of total cumulative counts of acute rejection was also assessed to see whether there was a stronger effect. There was a significant, but not stronger, impact on time to BOS $(\mathrm{RR}=1.2, p=0.009)$, but not on time to $\mathrm{OB}(\mathrm{RR}=0.9, p=0.31)$.

HLA mismatches. Mismatches at the A loci, as well as the total number of HLA mismatches, were found to be significant risk factors for the development of BOS. Mismatches at the B loci or the DR loci, when considered individually, were not found to have an effect on the development of BOS. Number of HLA mismatches was not associated with time to $O B$.

DASH test. In an experimental study, the DASH test was performed in 29 patients. No recipients with DASH have had a diagnosis of chronic lung dysfunction (2-year survival free from BOS, $100 \%$ vs $52 \%$; $p=0.005)$ or of histologically proved OB (2-year survival free from $\mathrm{OB}, 100 \%$ vs $52 \% ; p=0.01$ ).

Multivariate proportional hazards regression model based on clinical experience. A model was evaluated that included all variables thought to have value in predicting development of $\mathrm{BOS}$ or $\mathrm{OB}$ based on clinical experience, regardless of statistical association. That model included age older than 50 years, HLA mismatches at the A loci, primary pulmonary hypertension, steroid regimen, rejection episodes before 90 days, cumulative episodes of acute rejection, and CMV pneumonitis. Late acute rejection, CMV pneumonitis, and HLA mismatches at the A loci were associated with the development of BOS $(n=88$; Table IV). Repeated analysis without the HLA data $(n=121)$ had similar results. Only CMV pneumonitis was associated with the development of $\mathrm{OB}$.

Multivariate proportional hazards regression models based on statistical criteria. Using the variables specified in the clinical model, CMV pneumonitis, HLA mismatches at the A loci, and cumulative episodes of late acute rejection were significantly associated with the development of BOS $(n=88$; Table V). The only variable that was a significant predictor of time to $\mathrm{OB}$ was $\mathrm{CMV}$ pneumonitis $(\mathrm{RR}=2.3, p=0.02)$. Resulting models on the 121 patients excluding the HLA data had the same significant associations with similar relative risks.

\section{Discussion}

The major factor affecting the long-term survival after lung transplantation is chronic allograft dys- 
Table II. Assessment of individual fixed covariates for two end points by proportional hazards regression: Biopsy-proved $O B$ and $B O S$

\begin{tabular}{|c|c|c|c|c|c|c|}
\hline & \multicolumn{3}{|c|}{$B O S \geq 2$} & \multicolumn{3}{|c|}{$O B$} \\
\hline & $R R$ & $95 \% C I$ & $p$ Value & $R R$ & $95 \% \mathrm{CI}$ & $p$ Value \\
\hline \multicolumn{7}{|l|}{ Diagnostic group } \\
\hline \multicolumn{7}{|l|}{$\alpha_{1}-A T+$ COPD (ref grp) } \\
\hline PPH + Eisenmenger's & 0.96 & $0.48-1.9$ & 0.96 & 1.4 & $0.6-3.0$ & 0.44 \\
\hline Other & 1.1 & $0.49-2.4$ & 0.92 & 0.9 & $0.3-2.4$ & 0.78 \\
\hline PPH only & 1.2 & $0.6-2.3$ & 0.67 & 1.6 & $0.7-3.5$ & 0.23 \\
\hline \multicolumn{7}{|l|}{ Transplant type } \\
\hline \multicolumn{7}{|l|}{ Single lung (ref grp) } \\
\hline Bilateral single lung & 0.76 & $0.31-1.9$ & 0.55 & 1.1 & $0.4-3.1$ & 0.81 \\
\hline Heart-lung & 0.82 & $0.40-1.7$ & 0.58 & 1.2 & $0.5-2.6$ & 0.70 \\
\hline \multicolumn{7}{|l|}{ Age } \\
\hline \multicolumn{7}{|l|}{$<50$ yr (ref grp) } \\
\hline$>50 \mathrm{yr}$ & 0.96 & $0.5-1.8$ & 0.89 & 0.54 & $0.2-1.3$ & \\
\hline Sex: female & 1.0 & $0.6-1.8$ & 0.91 & 1.3 & $0.6-2.5$ & 0.51 \\
\hline Ischemic time & 1.0 & $0.9-1.1$ & 0.84 & 1.0 & $0.9-1.0$ & 0.40 \\
\hline $\begin{array}{l}\text { Steroid treatment } \\
\quad \text { (before 1991-ref grp) }\end{array}$ & 0.76 & $0.4-1.5$ & 0.42 & 0.7 & $0.3-1.5$ & 0.34 \\
\hline \multicolumn{7}{|l|}{ HLA mismatches } \\
\hline A loci & 1.8 & $1.0-3.2$ & 0.04 & 1.3 & $0.7-2.5$ & 0.43 \\
\hline B loci & 1.5 & $0.7-3.1$ & 0.26 & 1.1 & $0.5-2.5$ & 0.75 \\
\hline DR loci & 1.2 & $0.7-2.1$ & 0.44 & 1.1 & $0.6-2.1$ & 0.71 \\
\hline Total mismatches & 1.4 & $1.0-1.9$ & 0.04 & 1.1 & $0.8-1.6$ & 0.47 \\
\hline Days on waiting list & 1.0 & $0.9-1.0$ & 0.84 & 1.0 & $0.9-1.0$ & 0.19 \\
\hline $\begin{array}{l}\text { Rejection episodes } \\
\text { (first } 90 \text { days) }\end{array}$ & 1.2 & $0.9-1.6$ & 0.11 & 1.0 & $0.7-1.4$ & 0.83 \\
\hline
\end{tabular}

$\alpha_{1}-A T, \alpha_{1}$-Antitrypsin deficiency; $C I$, confidence interval; $C O P D$, chronic obstructive pulmonary disease; $P P H$, primary pulmonary hypertension; ref grp, reference group.

Table III. Assessment of time-dependent covariates for two end points by proportional hazards regression: Biopsy-proved $O B$ and BOS

\begin{tabular}{|c|c|c|c|c|c|c|}
\hline & \multicolumn{3}{|c|}{$B O S \geq 2$} & \multicolumn{3}{|c|}{$O B$} \\
\hline & $R R$ & $95 \% C I$ & $p$ Value & $R R$ & $95 \% C I$ & $p$ Value \\
\hline CMV pneumonitis & 3.2 & $1.8-5.8$ & 0.0001 & 3.6 & $1.8-7.3$ & 0.0005 \\
\hline $\begin{array}{l}\text { Total cumulative episodes } \\
\text { of acute rejection }\end{array}$ & 1.2 & $1.0-1.4$ & 0.009 & 1.1 & $0.9-1.3$ & 0.31 \\
\hline $\begin{array}{l}\text { Cumulative episodes of } \\
\text { late acute rejection }\end{array}$ & 1.3 & $1.0-1.5$ & 0.02 & 0.9 & $0.8-1.2$ & 0.55 \\
\hline PLT class II & 1.3 & $0.6-2.9$ & 0.49 & 1.7 & $0.7-4.4$ & 0.23 \\
\hline
\end{tabular}

function, thought to be synonymous with chronic rejection. This syndrome is a major cause of late morbidity and mortality for lung allograft recipients. The term BOS has been used to describe such dysfunction, with a staging system based on forced expiratory volume in 1 second, with or without pathologic evidence of OB. ${ }^{3}$ This system was designed to allow comparison of results between institutions owing to the low sensitivity of transbronchial biopsies in detecting $\mathrm{OB}^{13}$ and a high incidence of
OB not detected clinically but found at autopsy in patients with chronic lung dysfunction not attributed to other causes. ${ }^{2}$

$\mathrm{OB}$ is a progressive obstructive airway disease identified histologically by the presence of small airway inflammation and fibrosis of the lamina propria and lumen and characterized clinically by rapidly progressive airflow obstruction. OB was first reported after heart-lung transplantation in $1985 .{ }^{14}$ $\mathrm{OB}$ is a major cause of death and disability in 
Table IV. Multivariate proportional hazards model predicting $B O S$ and $O B$ based on clinical variables $(n=88)$

\begin{tabular}{llll}
\hline & $R R$ & $95 \% C I$ & $p$ Value \\
\hline BOS $\geq 2$ & & & \\
$\quad$ Acute rejection (<90 days) & 1.2 & $0.8-1.6$ & 0.40 \\
$\quad$ Cumulative episodes of acute & 1.2 & $1.0-1.5$ & 0.04 \\
$\quad$ rejection & & & \\
CMV pneumonitis & 3.4 & $1.7-6.8$ & 0.0005 \\
Age $>50$ yr & 0.9 & $0.4-2.0$ & 0.83 \\
HLA mismatches at A loci & 2.1 & $1.1-4.2$ & 0.03 \\
$\quad$ Primary pulmonary hypertension & 1.7 & $0.7-4.0$ & 0.20 \\
$\quad$ Steroid regimen after 1990 & 0.9 & $0.4-1.9$ & 0.70 \\
OB & & & \\
$\quad$ Acute rejection (<90 days) & 1.1 & $0.7-1.7$ & 0.63 \\
Cumulative episodes of acute & 0.9 & $0.7-1.1$ & 0.30 \\
$\quad$ rejection & & & \\
CMV pneumonitis & 2.4 & $1.1-5.5$ & 0.04 \\
Age $>50$ yr & 0.5 & $0.2-1.5$ & 0.24 \\
HLA mismatches at A loci & 1.2 & $0.6-2.3$ & 0.60 \\
$\quad$ Primary pulmonary hypertension & 1.3 & $0.5-3.4$ & 0.64 \\
Steroid regimen after 1990 & 0.6 & $0.2-1.7$ & 0.34 \\
\hline
\end{tabular}

long-term survivors of lung transplantation, occurring in up to $50 \%$ of patients surviving the first year after transplantation. ${ }^{15}$ Augmentation of immunosuppression has been advocated and appears to ameliorate or stabilize the disease process in some patients. ${ }^{16,17}$ Despite this, OB often progresses relentlessly, leading to death. Identifying risk factors for $O B$ is an important goal so that patients in whom the risk is high can receive close surveillance and early treatment. Risk factors identified by this analysis included CMV pneumonitis, acute rejection after 90 days, negative DASH test results, number of HLA mismatches at the A loci, and total number of HLA mismatches.

The immunopathogenic mechanisms leading to the development of $\mathrm{OB}$ are incompletely understood. An experimental large animal model of $\mathrm{OB}$ demonstrated increased lymphocyte counts with $\mathrm{CD} 8+$ cells predominating on BAL and increased expression of MHC class II antigens on the bronchiolar epithelium after the development of $\mathrm{OB} .^{18}$ Studies performed at our institution have identified BAL-derived lymphocytes demonstrating PLT reactivity that correlated with $\mathrm{MHC}$ antigen expression with stable or minimally progressive versus rapidly progressive OB. ${ }^{4}$ These studies demonstrated the role of augmented immunosuppressive therapy in halting or slowing this disease process in selected patients with PLT reactivity against class II antigens; by contrast, PLT reactivity against class I antigens
Table V. Multivariate proportional hazards model predicting $B O S$ and $O B$ based on stepwise regression

\begin{tabular}{lccc}
\hline & $R R$ & $95 \% \mathrm{CI}$ & $p$ Value \\
\hline $\begin{array}{l}\text { BOS } \geq 2 \\
\quad \text { Cumulative episodes of }\end{array}$ & 1.3 & $1.0-1.6$ & 0.02 \\
$\quad \begin{array}{l}\text { acute rejection } \\
\text { CMV pneumonitis }\end{array}$ & 3.2 & $1.7-6.2$ & 0.0006 \\
$\quad$ HLA mismatches at A loci & 2.4 & $1.3-4.6$ & 0.006 \\
OB $\quad$ & & & \\
CMV pneumonitis & 2.2 & $1.1-4.3$ & 0.02 \\
\hline
\end{tabular}

resulted in progressive $O B$ with all patients dying within 6 months of diagnosis. These results suggest that distinct immune modulation mechanisms are responsible for the subsequent development of $\mathrm{OB}$.

A decline in survival for patients with BOS was predominantly noted to occur 12 to 24 months after transplantation, with stabilization of long-term survival for those patients surviving beyond 36 to 48 months. Similar findings were noted in patients with or without histologic OB. These findings suggest the existence of two pathologic variants of chronic allograft dysfunction: one that is rapidly progressive and associated with death within 24 to 36 months and one that is chronically stable and associated with a better long-term survival.

Infections, including those caused by CMV, Pneumocystis carinii, and Ebstein-Barr virus, have all been implicated in the pathogenesis of OB. ${ }^{19}$ In this study, we found no difference between patients with and without chronic lung dysfunction with regard to isolation of $\mathrm{CMV}$ from BAL fluid obtained from symptom-free patients in stable condition. However, the development of CMV pneumonitis was significantly associated with chronic lung dysfunction. Duncan and associates ${ }^{20}$ have also shown that the prevalence of chronic rejection was highest among patients with CMV-positive results and with biopsy or cytology proved pneumonitis. In contrast, Cooper and colleagues ${ }^{1}$ reported that posttransplantation CMV infections did not influence the subsequent development of OB. The reasons for this discrepancy are not clear. On the basis of our results, theoretical approaches would include CMV matching between donor and recipient, as well as administration of prophylactic anti-CMV therapy in lung allograft recipients. However, we do not routinely perform CMV matching between donor and recipient, because this would cause further constraint on the availability of donor lungs. Prophylactic antiCMV treatment is now practiced by most lung 
transplant centers because of favorable results in patients undergoing lung, ${ }^{21}$ heart, ${ }^{22}$ and bone marrow $^{23}$ transplantation. Further studies will need to be carried out to determine the optimal drug regimen and dosing schedule.

Acute rejection is a common occurrence in lung allograft recipients. In this study we did find a correlation between the cumulative count of rejection episodes and the subsequent development of chronic lung dysfunction. Experimental lung transplant models have shown the influence of acute rejection on the subsequent development of chronic rejection. ${ }^{18,24}$ Clinical studies have also implicated acute rejection as a significant risk factor for $\mathrm{OB} .^{25}$ The intensity and the persistence of early acute rejection have been correlated with the development of $\mathrm{OB},{ }^{26,27}$. prompting early detection and treatment of acute rejection followed by increased maintenance immunosuppression to achieve normal biopsy results. ${ }^{28}$

The concept that increased immune activation is responsible for $\mathrm{OB}$ is based on the reactivity of specific cells in the lung allograft against donor antigens. BAL-derived cells exhibit donor antigenspecific alloreactivity in OB when tested in PLT and cell-mediated lympholysis assays. ${ }^{29}$ Studies at the University of Pittsburgh have shown a correlation between a positive PLT of BAL cells from patients with a negative biopsy and the development of $\mathrm{OB}$ on a subsequent biopsy. ${ }^{30,31}$ Studies from the University of Minnesota have focused on the other end of the spectrum of donor-specific reactivity to further add evidence to the immunologic basis of chronic allograft rejection. ${ }^{7}$ Specifically, the development of DASH in renal transplant recipients correlated with an improved overall graft outcome, as evidenced by fewer late rejection episodes and a decreased incidence of chronic rejection. ${ }^{32}$ In this study the development of DASH is associated with a better long-term outcome. Chronic lung dysfunction did not develop in any of our patients who had positive results from the DASH test; by contrast, chronic lung dysfunction developed in all patients whose DASH test results were negative. Thus DASH appears to be a useful test to predict a subpopulation of lung allograft recipients in whom OB is less likely to develop. However, further studies of the DASH test involving a larger population of lung allograft recipients is needed for confirmation of these findings. If confirmed, the DASHpositive subgroup could also be considered for a reduction in the immunosuppressive therapy admin- istered. The corollary could also be considered, that is, prophylactic augmentation of immunosuppression for patients whose DASH test results are negative.

In conclusion, this study indicates that patients who have CMV pneumonitis or who have more episodes of late acute rejection appear to be at high risk for chronic lung dysfunction after lung transplantation. Negative DASH test results and HLA mismatching were also identified as risk factors. Our findings suggest the following approaches to patient management. First, prophylactic therapy for CMV could reduce the prevalence of chronic lung dysfunction by reducing the frequency of CMV pneumonitis. Second, increased focus on DASH may allow modification of immunosuppression on the basis of the DASH test response. Regular surveillance biopsies in these patients, combined with a high index of suspicion, may facilitate an early diagnosis of chronic allograft dysfunction. However, it remains to be seen whether an early diagnosis of chronic lung dysfunction with subsequent therapy will improve the outcome of these patients.

\section{REFERENCES}

1. Cooper JD, Patterson GA, Trulock EP, the Washington University Lung Transplant Group. Results of single and bilateral lung transplantation in 131 consecutive recipients. J Thorac Cardiovasc Surg 1994;107:460-71.

2. Sundaresan S, Trulock EP, Mohanakumar T, Cooper JD, Patterson GA. Prevalence and outcome of bronchiolitis syndrome after transplantation. Ann Thorac Surg 1995;60: 1341-7.

3. Cooper JD, Billingham M, Egan T, et al. A working formulation for the standardization of nomenclature and clinical staging of chronic dysfunction in lung allografts. J Heart Lung Transplant 1993;12:713-6.

4. Reinsmoen NL, Bolman RM, Savik K, Butters K, Hertz MI Are multiple immunopathogenetic events occurring during the development of obliterative bronchiolitis and acute rejection? Transplantation 1993;55:1040-4.

5. Bolman RM, Shumway SJ, Estrin JA, Hertz MI. Lung and heart-lung transplantation: evolution and new applications. Ann Surg 1991;214:456-70.

6. Yousem SA, Berry GJ, Brunt EM, et. al. A working formulation for the standardization of nomenclature in the diagnosis of heart and lung rejection: lung rejection study group. J Heart Transplant 1990;9:593-601.

7. Reinsmoen NL, Bolman RM, Savik K, Butters K, Matas AJ, Hertz MI. Improved long-term graft outcome in lung transplant recipients who have donor antigen-specific hyporeactivity. J Heart Lung Transplant 1994;13:30-7.

8. Kalbfleisch JD, Prentice RL. The statistical analysis of failure time data. New York: John Wiley; 1980. p. 70-117.

9. Allison PD. In: Allison PD, editor. Survival analysis using the SAS system: a practical guide. Cary (NC). SAS Institute, Inc., 1996:111-83. 
10. Kalbfleisch JD, Prentice RL. The statistical analysis of failure time data. New York: John Wiley; 1980. p. 122-40.

11. Kaplan EL, Meier P. Nonparametric estimation from incomplete observations. J Am Stat Assoc 1958;53:457-81.

12. Kshettry VR, Kroshus TJ, Savik K, Hertz MI, Bolman RM. Primary pulmonary hypertension as a risk factor for the development of obliterative bronchiolitis in lung allograft recipients. Chest 1996;110:704-9.

13. Kramer MR, Stoehr C, Whang JL, et al. The diagnosis of obliterative bronchiolitis after heart-lung and lung transplantation: low yield of transbronchial lung biopsy. J Heart Lung Transplant 1993;12:675-81.

14. Burke CM, Morris AJ, Dawkins KD, et al. Late air flow obstruction in heart-lung transplant recipients. Heart Transplant 1985;4:437-40.

15. Burke CM, Theodore J, Baldwin JC, et al. Twenty-eight cases of human heart-lung transplantation. Lancet 1986;1:517-9.

16. Griffith BP, Paradis IL, Zeevi A, et al. Immunologically mediated disease of the airways after pulmonary transplantation. Ann Surg 1988;208:371-8.

17. Glanville AR, Baldwin JC, Burke CM, et al. Obliterative bronchiolitis after heart-lung transplantation: apparent arrest by augmented immunosuppression. Ann Intern Med 1987; 107:300-4.

18. Al-Dossari GA, Kshettrey VR, Jessurun J, Bolman RM. Experimental large-animal model of obliterative bronchiolitis after lung transplantation. Ann Thorac Surg 1994;58:34-40.

19. Paradis I, Dummer JS, Dauber J, et al. Risk factors for the development of chronic rejection of the human lung allograft. Am Rev Respir Dis 1989;139:A529.

20. Duncan AJ, Duman JS, Paradis IL, et al. Cytomegalovirus infection and survival in lung transplant recipients. J Heart Lung Transplant 1992;10:638-46.

21. Duncan SR, Paradis IL, Dauber JH, Youssem SA, Hardesty RL, Griffith BP. Ganciclovir prophylaxis for cytomegalovirus infections in pulmonary allograft recipients. Am Rev Respir Dis 1992;146:1213-5.

22. Merigan TC, Renlund DG, Keay S, et al. A controlled trial of ganciclovir to prevent cytomegalovirus disease after heart transplantation. N Engl J Med 1992;326:1182-6.

23. Goodrich JM, Bowden RA, Fisher L, Keller C, Schoch G, Meyers JD. Ganciclovir prophylaxis to prevent cytomegalovirus disease after allogenic marrow transplantation. Ann Intern Med 1993;118:173-8.

24. Hertz MI, Jessurun J, King MB, Savik K, Murray JJ. Reproduction of the obliterative bronchiolitis lesion after heterotopic transplantation of mouse airways. Am J Pathol 1993; 142:1945-51.

25. Bando K, Paradis IL, Similo S, et al. Obliterative bronchiolitis after lung and heart-lung transplantation. J Thorac Cardiovase Surg 1995;110:4-14.

26. Yousem SA, Dauber JA, Keenan R, Paradis IL, Zeevi A, Griffith BP. Does histologic acute rejection in lung allografts predict the development of bronchiolitis obliterans? Transplantation 1991;52:306-9.

27. Clelland C, Higgenbottam TW, Oyulana B, et al. Histologic prognostic indicators for lung allografts of heart-lung transplants. J Heart Lung Transplant 1990;9:177-86.

28. Scott JP, Higgenbottam TW, Sharples L, et al. Risk factors for obliterative bronchiolitis in heart-lung transplant recipients. Transplantation 1991;51:813-7.

29. Reinsmoen NL, Bolman RM, Savik K, Butters KL, Hertz MI. Differentiation of Class I-- and Class II-directed donor-specific alloreactivity in bronchoalveolar lavage lymphocytes from lung transplant recipients. Transplantation 1992;53:181-9.

30. Rabinowich $\mathrm{H}$, Zeevi A, Yousem SA, et al. Alloreactivity of lung biopsy and bronchoalveolar derived lymphocytes from pulmonary transplant patients: correlation with acute rejection and bronchiolitis obliterans. Clin Transplant 1990;4:376-84.

31. Zeevi A, Rabinowich $\mathrm{H}$, Yousem SA, et al. Presence of donor-specific alloreactivity in histologically normal lung allografts is predictive of subsequent bronchiolitis obliterans. Transplant Proc 1991;23:1128-9.

32. Reinsmoen NL, Matas AJ. Improved late renal transplant outcome correlates with the development of in vitro antigenspecific reactivity. Transplantation 1993;55:1017-23. 